\title{
Gesundheitsbezogene Daten und Proben für die Forschung
}

Das Humanforschungsgesetz (HFG) lässt unter bestimmten Voraussetzungen einen sogenannten Generalkonsent (GK) zu, mit dem Personen in die Verwendung ihrer Daten und Proben für künftige Forschungsprojekte einwilligen können. Einige Schweizer Spitäler haben den GK bereits eingeführt, die Informations- und Einwilligungsformulare variieren jedoch. Dies erschwert die Forschungszusammenarbeit über Institutionen hinweg. Die Schweizerische Akademie der Medizinischen Wissenschaften (SAMW) und die Dachorganisation der Schweizer Ethikkommissionen für die Forschung am Menschen (swissethics) stellen nun eine Vorlage GK Schweiz zur Verfügung. Diese besteht aus einer Kurzfassung (inkl. Einwilligung) und einer weiterführenden Begleitinformation (Broschüre).

Die Vorlage wurde von einer Arbeitsgruppe ${ }^{1}$ ausgearbeitet, begleitend stellte die Swiss Biobanking Platform (SBP) Guidelines zur Verfügung, die den Umgang mit Daten und Proben standardisieren. ${ }^{2}$ Ebenfalls in den Ausarbeitungsprozess der Vorlage eingebunden waren Vertreterinnen und Vertreter von Patientenorganisationen, Spitälern und Forschung.

\section{Drei Grundsatzentscheide im Überblick}

1. Einwilligung für die Entnahme einer Probe für die Biobank als Option

Die Vorlage deckt die Verwendung von verschlüsselten genetischen und nicht-genetischen Daten und Proben ab, die routinemässig aus Diagnostik und Behandlung anfallen. Integriert wurde zudem die optionale Möglichkeit, mit dem GK die Einwilligung für eine zusätzliche Entnahme von Blut, Speichel oder Urin für Forschungszwecke einzuholen.
2. Verschlüsselte nicht-genetische Daten: Einwilligung statt Widerspruch

Das HFG (Art. 33 Abs. 2) erlaubt die Weiterverwendung von verschlüsselten nicht-genetischen gesundheitsbezogenen Daten ohne Einwilligung, sofern kein Widerspruch vorliegt. Die Vorlage GK Schweiz geht über das HFG hinaus und fordert auch für die Verwendung von verschlüsselten nicht-genetischen gesundheitsbezogenen Daten eine Einwilligung.

3. Umgang mit Widerruf und Zufallsbefunden Die Vorlage geht davon aus, dass im Falle eines Widerrufs Daten und Proben nicht mehr für neue Forschungsprojekte verwendet werden dürfen; auch nicht in anonymisierter Form. Im Umgang mit Zufallsbefunden lässt die Vorlage einen Ermessensspielraum offen. Sie geht davon aus, dass eine Information umso eher erfolgen muss, je schwerer die (bestehende oder zu erwartende) Krankheit ist, je gesicherter der konkrete Befund ist und je wahrscheinlicher eine effektive Therapieoption oder Präventionsmassnahme zur Verfügung steht.

\section{Vernehmlassung auf der SAMW-Website}

Der Entwurf der Vorlage GK Schweiz steht bis zum 31. März 2017 in einer öffentlichen Vernehmlassung. Interessierte Organisationen und Personen sind zur Stellungnahme eingeladen: samw.ch/vernehmlassung-2017-03

Die definitive Fassung der Vorlage GK Schweiz wird von swissethics verabschiedet und im April 2017 veröffentlicht. Für Kinder und Jugendliche und deren Eltern werden zeitnah spezifische Vorlagen bereitgestellt. 